\title{
HACIA UN PERFIL SOCIOLINGÜÍSTICO E HISTÓRICO DE LAS LENGUAS INDÍGENAS DE TABASCO
}

\author{
TOWARDS A SOCIOLINGUISTIC AND HISTORICAL PROFILE OF THE \\ INDIGENOUS LANGUAGES OF TABASCO
}

\author{
Amelia ESCOBAR POTENCIANO ${ }^{1}$ \\ Felipe Javier GALÁN LÓPEZ ${ }^{2}$
}

\begin{tabular}{|lll|}
\hline Recibido & $:$ & 29.11 .2020 \\
Aceptado & $:$ & 08.02 .2021 \\
Publicado & $:$ & 12.02 .2021 \\
\hline
\end{tabular}

\begin{abstract}
RESUMEN: El artículo muestra un perfil sobre las agrupaciones lingüísticas que existen en Tabasco, una pequeña entidad federativa situada en el sureste de México. Esto a partir de que la existencia de trabajos que aborden este tema es magra por lo que, con esta investigación situada bajo un abordaje teórico desde la sociolingüística, damos a conocer la importancia que tienen las lenguas indígenas en esta área en relación con las principales problemáticas de salud, educación y desarrollo. De ahí que se profundice más en estos datos, con el objetivo de contar con una descripción puntual y sustancial de la situación que enfrentan, presentando información sobre aspectos geográficos, sociales, económicos y lingüísticos, que toman como parteaguas, un necesario recuento histórico. Para esto, se siguieron las fórmulas propuestas por Lastra (1992), y la historiografía lingüística, así como información de bases de datos nacionales (INEGI, CONEVAL) que fueron complementados con un trabajo de investigación de campo realizado de 2016 a 2018 por diversas zonas indígenas de Tabasco.
\end{abstract}

Palabras clave: Lenguas indígenas, pueblos originarios, Tabasco, historia, perfil sociolingüístico.

\begin{abstract}
This paper shows a profile about those linguistic groups living in the state of Tabasco, located at southeast of Mexico. This due to the lack of works addressing this problem and, with this research with a theoretical approach from sociolinguistics, we discuss about the importance of indigenous languages in Tabasco in relation to the main problems of health, education and development. Hence, the present work delves deeper into these data, with the aim of having a punctual and substantial description of the situation faced by the native people of Tabasco, presenting information on geographical, social, economic and linguistic aspects, starting with a necessary historical count. We took formulas proposed by Lastra (1992) and linguistic history were followed, as well as information from national databases (INEGI, CONEVAL) in addition to the field work carried out from 2016 to 2018 by various indigenous areas of Tabasco.
\end{abstract}

Keywords: Indigenous languages, native peoples, Tabasco, history, sociolinguistic profile.

\footnotetext{
${ }^{1}$ Maestra en Lingüística. Universidad Intercultural del Estado de Tabasco. Estado de Tabasco-México. Email: amelia.escobar@uiet.edu.mx. ORCID: https://orcid.org/0000-0001-9608-7822

${ }^{2}$ Doctor en Historia y Estudios Regionales. Universidad Intercultural del Estado de Puebla. Estado de Puebla-México. Email: felipe.galan@uiep.edu.mx. ORCID: https://orcid.org/0000-0001-9715-2593
} 


\section{Journal of the Academy $|206|$}

\section{INTRODUCCIÓN}

En México existen 11 familias lingüísticas que concentran 68 agrupaciones y 364 variantes hasta ahora reconocidas y registradas de acuerdo a lo planteado por el Instituto Nacional de Lenguas Indígenas (INALI). En este estudio que trata sobre Tabasco, se puede encontrar representación de cuatro de estas familias y de siete agrupaciones lingüísticas, distribuidas a lo largo de sus 17 municipios integrados en dos regiones, con sus cinco subregiones denominadas como: la Chontalpa, el Centro, la Sierra, los Pantanos y los Ríos.

Muy poco se sabe sobre las características propias de cada una de estas lenguas en su variante tabasqueña, por lo que se desconoce también el efecto e impacto social que esto implica, entre varias razones consideramos que se debe a que esta entidad no fue prioritaria para las instituciones estatales del siglo XX como lo indica Brice (1986, 242-243) "La política del lenguaje en México, las entidades federativas que el INI consideró como prioritarias en base a la categoría de lengua y raza durante varias décadas fueron: Chiapas, Guerrero, Hidalgo, Estado de México, Michoacán, Oaxaca, Puebla, San Luis Potosí, Veracruz y Michoacán”.

A principio de siglo XXI, es de destacar que la nueva relación entre el Estado y sus pueblos indígenas, ha mostrado que en Tabasco sí existe una población indígena importante, por lo que hay que conocer sus lenguas, variantes regionales y locales, así como los impactos sociales en salud, economía, educación y desarrollo. A partir de lo anterior mostramos un panorama general de estas agrupaciones lingüísticas, presentando información en cuanto a su localización, situación sociolingüística y aspectos socioeconómicos, que permitirán al lector abrir la puerta hacia un conocimiento más vasto de estas culturas.

\section{DESARROLLO}

\section{Metodología}

El estudio propuesto es de tipo exploratorio y descriptivo, bajo los postulados teóricos de la sociolingüística y la historiografía lingüística. Parte de una revisión histórica para entender la importancia que tienen en Tabasco las lenguas indígenas en el tiempo. Para el alcance de los objetivos planteados presentamos una metodología mixta en las distintas fases de la 


\section{Journal of the Academy | 207 |}

investigación. La primera parte trata sobre un recuento histórico, posteriormente dos etapas, la primera heurística y hermenéutica, a fin de entender bajo una reflexión histórica a los pueblos indígenas que han existido en el territorio tabasqueño. Durante esta se realizó el acopio de las fuentes disponibles y el análisis de las mismas siguiendo dos categorías de análisis: identidad cultural histórica y lenguas registradas en Tabasco a través del tiempo.

En la segunda etapa, se muestra el resultado de trabajo de campo (2016-2018) durante las visitas a comunidades indígenas ch'oles, zoques y tzotziles, que son las que tienen una fuerte presencia histórica desde hace varios siglos, para recopilar información a través de técnicas de observación y entrevistas estructuradas, dicha información la mostramos de manera general. Las categorías de análisis se ampliaron y quedaron distribuidos en este trabajo de la siguiente forma:

1) Recuento histórico-lingüístico, 2) situación sociolingüística actual, 3) localidades indígenas, 4) aspectos socioeconómicos, 5) perfil sociolingüístico, 6) En la última parte presentamos las conclusiones y posicionamientos finales

Este estudio se enriqueció con información de bases de datos nacionales (Instituto Nacional de Estadística y Geografía (INEGI) y el Consejo Nacional de Evaluación de la Política de Desarrollo Social (CONEVAL), para elaborar el perfil sociolingüístico de hablantes de lenguas indígenas que actualmente habitan en Tabasco. Colateralmente, permitió la elaboración de la reseña histórica de las mismas a partir de la época prehispánica hasta el presente. El artículo expuesto es parte de las investigaciones del trabajo sociolingüístico de Amelia Escobar Potenciano en la Universidad Intercultural de Tabasco entre 2016-2018, y forma parte de los resultados de investigación histórica de Felipe Javier Galán López de la Universidad Intercultural de Puebla, durante su estancia posdoctoral en el año 2018-2019.

\section{i. Recuento histórico- lingüístico en Tabasco}

El actual territorio de Tabasco, cuenta con un pasado histórico muy extenso, pero poco explorado, debido a que sus fuentes de información se han extraviado en su mayoría o fueron destruidos por diferentes factores como el clima, las inundaciones, las incursiones de piratas 


\section{Journal of the Academy $\mid 208$ |}

en los siglos XVI y XVII, las luchas por el poder, la destrucción de archivos, por mencionar algunas (Martínez Assad, 2011).

En el año de 1579, hubo un registro fundamental para la historiografía de Tabasco, fue hecho por Melchor Alfaro y Santacruz y lo plasmó en las Relaciones históricos-geográficas de la provincia de Tabasco (Salazar, 2014), en base a ello podemos saber que las lenguas habladas en el antiguo territorio eran: el náhuatl, popoluca, chontal y zoque, esto además lo podemos encontrar en la obra de Ruz, 1991 y 2014. En los siguientes años de la colonia, fue el chontal la lengua más hablada en el área. Contaba con diversidad de vocablos y los conquistadores europeos la consideraron como un dialecto del maya.

Los principales intentos por conocer la presencia histórica de los indígenas en Tabasco, sucedieron durante el siglo XIX y principios del XX, cuando cronistas locales se dieron a la tarea de documentarlo. Destacan por su importancia los trabajos del Dr. Manuel Gil y Sáenz y el Dr. Mestre Ghigliazza, quienes hicieron grandes esfuerzos por ordenar la poca información lingüística que encontraron (Galán y Navarro, 2020).

Ya en el siglo XX hubo varios esfuerzos muy notables para estudiar la historia indígena y de las lenguas que se han hablado en Tabasco, por ejemplo, los cronistas locales como José Narciso Rovirosa, Bernardo del Águila y algunos más, dejaron valiosos documentos que pueden consultarse en las diferentes bibliotecas locales. No obstante, cabe destacar que durante las primeras décadas, los hallazgos y descubrimientos arqueológicos olmecas en la zona arqueológica de La Venta en el municipio de Huimanguillo, administrada por el Instituto Nacional de Antropología e Historia, fueron determinantes para la narrativa histórica que desde entonces se escribió sobre Tabasco: la de la civilización olmeca y la del nacionalismo indígena, que provocó además que se eliminara gradualmente de los censos, una gran parte del pasado histórico indígena y lingüístico de otras de las culturas que existen en Tabasco, que hablan las lenguas yokot'an, ch’ol y zoque. Esto tuvo como consecuencia que además en el imaginario de los tabasqueños existiera una imposición del mundo olmeca de museo, sobre el resto de las culturas de origen mesoamericano que se asentaron en Tabasco desde varios siglos atrás. (Galán y Navarro, 2020). 


\section{Journal of the Academy $|209|$}

Los trabajos arqueológicos fueron determinantes, no así la parte etnográfica y lingüística que fue poco trabajada en Tabasco, los recuentos historiográficos de Lorenzo Ochoa en 1988, sobre los cien años de la antropología e historia tabasqueña son un referente central, además de las investigaciones de Carlos Incháustegui, Mario Humberto Ruz, Flora Salazar, entre otros. La presencia de pueblos herederos de las civilizaciones mesoamericanas en Tabasco, resultan esenciales para entender cómo perduraron hasta la actualidad las distintas lenguas originarias con sus variantes.

Algunos investigadores (Rivet 1954, De Vos 1980, Ciudad y Lacadena 2001) refieren que los primeros pobladores entraron a Tabasco desde la parte noroccidental y se asentaron sobre la orilla de ríos entre los años 1050 y 900 a. c. Estos asentamientos fueron dependientes de los sitios de La Venta Tabasco y San Lorenzo en el sur de Veracruz, con los que mantenían relaciones comerciales estrechas. Durante el siglo XIX y XX se realizaron varias investigaciones arqueológicas e históricas que dan cuenta de ello.

Posteriormente llegaron los mayas a Tabasco. Algunos autores mencionan que ingresaron a esta área alrededor del año 250 d. C., para ocupar sitios como El Tortuguero, Arenal, Reforma y algunos más. Otros indican que entraron por la Chontalpa desde la región de Palenque, Valle del Tulijá y medio Usumacinta, alrededor del año 200 a.C. (West et al 1969). También existe la creencia que es narrada por cronistas locales de que llegaron de la isla de Cozumel a estos territorios, encabezados por un personaje llamado Auxahual estableciéndose en la parte noreste tabasqueña.

Hay referentes como los de Scholes y Roys (1996), para asegurar que los antiguos indígenas tabasqueños eran de habla maya-chontal y que los mayas de Yucatán se referían a ellos llamándolos puntunes o punctunes. En las inscripciones mayas de Acalán, se le llama a esta lengua uiba tan 'la lengua de aquí'. Hay evidencia arqueológica que indica que ellos fundaron la ciudad de Acalán y que después de asentarse, este grupo maya-chontal comenzó a expandirse territorialmente, conquistando a las poblaciones aledañas a su asentamiento (Shholes y Roys 1996). Uno de estos pueblos conquistados fueron las poblaciones que habitaban la región de Tadnoziq (Tenosique al sur de Tabasco) según lo refieren los registros paleográficos de Smalius: 


\section{Journal of the Academy $|210|$}

Unacahibal auxaual ta/i cuçumil tali uchuci cabil cabob vi koti umole cah tanodzic yithoc unucalob huncha yithoc paxoc yithoc chacbalam uchantulib paxmulu hainix ulotob. Ulachuci cabob. En su principio está Auxaual; vino de Cozunlel; vino y capturó la tierra y los pueblos; llegó aquí a juntar el pueblo de Tanodzic, con sus grandes, Huncha, Paxoc, Chacbalam y el cuarto Paxmulu; éstos fueron sus acompañantes. El capturó las tierras completamente. (Ciudad y Lacadena, 2001, p. 18-19)

Los antepasados de los actuales yokot'anes, conocidos también como chontales, estaban situados en las márgenes de los ríos Usumacinta y Grijalva, esta ocupación estratégica y el dominio de la región, les permitió el liderazgo comercial de la zona. Esta situación estuvo relacionada con fuertes influencias extranjeras del centro de Mesoamérica, sobre todo al náhuatl, de tal forma que eran casi bilingües.

De hecho, la palabra chontal proviene de la voz náhuatl chontalli que significa 'extranjero' y fue utilizada por esta cultura para designar a los pueblos que habitaban esta región. Se habla de una gran inmigración nahua hacia esta zona que, en un primer estado, influenció la cultura y la lengua de los putunes debido a su prestigio como lengua franca y como nación dominante. Por sus relaciones comerciales, se cree que estos grupos putunes llevaron la influencia náhuatl hasta la península de Yucatán. (Maza, 2012, p. 17)

Para confirmar la presencia del ch'ol en la región, citamos a Alejos y Martínez (2007) quienes relatan que los ch'oles habitaban en la parte sur del Petén, en Palenque Chiapas y en Tabasco a la llegada de los españoles, junto con hablantes de otras lenguas que también se separaron del maya. De hecho, las lenguas ch'ol, chortí y chontal, están emparentadas y conforman el grupo cholano. Esto indica que hay coincidencia entre los elementos léxicos y gramaticales entre ellos (Schumann, 1973).

El grupo cholano es considerado por los arqueólogos como la lengua de prestigio de las inscripciones mayas de los sitios ceremoniales, ha sido utilizada para intuir, por ejemplo, que la antigua ciudad de Palenque estuvo habitada por ch'oles. Los arqueólogos han propuesto la hipótesis que las lenguas tienen que ver con las antiguas inscripciones, donde sus antiguos habitantes dejaron elementos propios de su morfología, léxico y sintaxis. Así que esta evidencia apunta que la lengua maya se mezcló con una lengua minoritaria, para dar como resultado un dialecto maya que más tarde alcanzó el estatus de lengua (Houston et al, 1998; Voss 2018). 


\section{Journal of the Academy $|211|$}

Durante todo el siglo XX hubo una migración de grupos ch'oles principalmente de la región norte del vecino estado de Chiapas hacia Tabasco, principalmente hacia su región montañosa, como lo refieren Ruz (2014), Rojas (2019), Escobar (2019) y Sosa (2019), entre otros.

Los mayas chontales tenían como sitios importantes a Copilco, mientras que en la ribera del río Grijalva (el más importante de Tabasco), se encontraba Potonchán, la ciudad más habitada por chontales según registros arqueológicos existentes, además de Chilapa y Tepetitán. También se considera que había pueblos mayas hacia la Sierra, los llamados zaguatanes (Astapa, Jahuacapa y Jalapa) (Ruz, 1991)

La segunda lengua más hablada por muchos años en Tabasco fue el náhuatl, sus habitantes se asentaron en sitios como Zimatlan y Xicalango, así como en Cunduacán y Cualquiteupa. Los naguatanes de Huimango y los ahualules también se consideran parte de esta etnia. Aunque sus poblaciones eran de poco número, esta lengua todavía conserva un prestigio alto.

Por último, encontramos a los zoques. Esta población en un primer momento ocupó la región norte de la chontalpa (Cimatan), pero migraron hacia el sur del territorio. En esta área fundaron los pueblos de Tacotalpa, Teapa, Tecomajiaca, Tapijulapa, Osolotlan (hoy Oxolotán) y Puxcatan, en una región cultural compartida con los zoques de Chiapas (Ruz, 2014) Wiliamson en un estudio histórico y lingüístico, afirmó lo siguiente:

El origen del nombre de Tabasco es oscuro y debatido, aunque es claramente indígena. Bernal Díaz del Castillo apunta por ejemplo en el capítulo 30 de su Historia verdadera de la conquista de la Nueva España, que "en tres días que navegamos llegamos al río Grijalva que es nombrado en lengua de indios de Tabasco. Desafortunadamente no da ningún indicio de que lengua hablaban y no sabemos si los nativos aplicaban el nombre del territorio además del río y del pueblo." (Williamson, 1986, p. 54).

Precisamente este autor es muy claro en recordar que fue Rovirosa en 1888, quien hizo un esfuerzo por explicar las diferentes lenguas que se hablaron en Tabasco en el pasado, dividiendo por partidos la presencia indígena. La presencia chontal era en el siglo XIX la más importante en Tabasco, y así ha sido hasta el presente, pero resulta importante mencionar que retomando los registros de los cronistas como Rovirosa y que analizó Williamson, la presencia indígena ha sido determinada además de los chontales, por los grupos mayas y nahuas. 
...dejando de lado el ch'ol, cuya vigencia en territorio tabasqueño depende de un pequeño grupo de inmigrantes de Chiapas, se hablaban en Tabasco 5 lenguas, pertenecientes dos familias lingüísticas: los grupos maya y náhuatl....Según Rovirosa las lenguas nahuas, el mexicano y al ahualulco se hablaban en la parte occidental del estado, el zoque en el sur y el chontal se extendía por todo el estado...Étnica y culturalmente, los orígenes de los chontales se pierde en un pasado desconocido y misterioso. Su nombre deriva del náhuatl chontalli, forastero, extranjero... (Williamnson, 1986, p. 62-63).

La presencia de población de origen mesoamericano durante la colonia, pasó por varios procesos históricos que son determinantes para conocer la situación sociolingüística actual de Tabasco. En el siglo XVI las epidemias que azotaron al sureste mexicano, diezmaron a la población originaria como sucedió en otras partes de México, los cálculos refieren un 94\% de las poblaciones originarias que murieron entre 1520 y 1579 (Martínez Assad, 2011).

La construcción histórica del territorio tabasqueño fue larga, pero su denominación tuvo que ver, según Rovirosa por la construcción de un vocablo indígena, afirma Williamson, (1986: 56): "Aqueste río se llama de Tabasco porque el cacique de aquel pueblo se decía Tabasco". Tlalli y paltic, significaban tierra mojada, y que Taabscob, hacía referencia a la caída de Mayapan".

Resulta muy interesante revisar los trabajos de los cronistas tabasqueños, quienes trataron de explicar la conformación histórica de Tabasco en base a su pasado lingüístico. Por ejemplo, Rovirosa, en 1888, hablaba de esta tierra como zona de transición lingüística, donde confluyeron varias lenguas.

El siglo XX fue determinante para el desarrollo de una política lingüística en México y que impactó en la política en Tabasco, la cual fue implementada entre 1940 y que se extendió hasta el fin de siglo. Esta tuvo que ver con las instituciones indigenistas como el INI entre 1940 y 1990 y después ya en un nuevo siglo fue creada la CDI (Comisión Nacional para el Desarrollo de los Pueblos Indígenas) en el año 2003 y recientemente el Instituto Nacional de los Pueblos Indígenas (INPI desde 2018 hasta el presente.

Los registros de los censos durante el siglo XX, pueden dar cuenta de cómo la política indigenista de siglo XX, no miró con atención a los pueblos indios tabasqueños, y es por eso que a principio de siglo XXI, la población que las instituciones como la CDI, el INEGI o 


\section{Journal of the Academy $|213|$}

Coneval en Tabasco, registran como población indígena es muy poca (alrededor de 3.7\%). Aunque como varios investigadores lo están expresando, existe mucha población tabasqueña que sí se auto adscribe como ch'ol, yokot'an y zoque, de ahí la importancia de este estudio.

\section{ii. Situación sociolingüística actual}

Como se observa en la tabla 1, en cada uno de los 17 municipios que integran Tabasco hay presencia de población indígena con herencia de un pasado histórico; el criterio de HLI (Hablantes de Lenguas Indígenas) que utiliza el INEGI desde hace varios años y que es producto de los censos de principio de siglo XX, sigue siendo esencial para definir a la población étnicamente diferenciada. Algunas de estas poblaciones originarias, han habitado tanto tiempo en Tabasco que se desconoce una fecha exacta de su origen, pero hay muchas otras provenientes de entidades distintas, que se establecieron en el actual territorio tabasqueño.

\section{Tabla 1:}

Familias y agrupaciones lingüísticas que residen en Tabasco

\begin{tabular}{|c|c|c|c|}
\hline Familia & Agrupación & $\begin{array}{l}\text { Número de } \\
\text { localidades }^{3}\end{array}$ & Municipio \\
\hline \multirow[t]{5}{*}{ Maya } & $\begin{array}{l}\text { Chontal o } \\
\text { yokot'an }\end{array}$ & 164 & $\begin{array}{l}\text { Balancán, Centla, Centro, Cunduacán, } \\
\text { Jalpa de Méndez, Jonuta, Nacajuca, } \\
\text { Macuspana }\end{array}$ \\
\hline & Ch'ol & 97 & $\begin{array}{l}\text { Balancán, E. Zapata, Macuspana, } \\
\text { Nacajuca, Centro, Tacotalpa y Tenosique }\end{array}$ \\
\hline & Tzeltal & 17 & Balancán, E. Zapata, Jonuta y Tenosique \\
\hline & Tzotzil & 3 & Huimanguillo, Teapa y Tacotalpa \\
\hline & Maya & 1 & Cárdenas \\
\hline Mixezoque & Zoque & 7 & Huimanguillo, Jalpa y Tacotalpa \\
\hline Otomangue & Zapoteco & 1 & Cunduacán \\
\hline Yutoazteca & Náhuatl & 1 & Comalcalco \\
\hline
\end{tabular}

Nota: INEGI (2010).

\footnotetext{
${ }^{3}$ Localidades con presencia de población indígena o con población indígena dispersa 


\section{Journal of the Academy $|214|$}

El total de hablantes de estas lenguas en 2010 se calculó en 60526 personas que, a su vez, representan el 3\% de la población total (INEGI, 2010). Esta cifra coloca al estado por debajo del porcentaje determinado como media nacional. Sin embargo, los Indicadores Socioeconómicos de los Pueblos Indígenas de México (2015:112), calcularon un total de 122397 hablantes para 2015. La proporción de HLI por municipios que se observa en este reporte puede apreciarse en la tabla 2.

\section{Tabla 2:}

Población total e indígena de 3 años y más en Tabasco

\begin{tabular}{|c|c|c|c|c|}
\hline Municipio & Población total & $\begin{array}{c}\text { Población } \\
\text { indígena }\end{array}$ & $\%$ & Tipo \\
\hline Tacotalpa & 48784 & 18959 & 38.9 & $\mathrm{C}$ \\
\hline Centla & 110130 & 12754 & 11.6 & $\mathrm{C}$ \\
\hline Nacajuca & 138366 & 243283 & 10.5 & $\mathrm{C}$ \\
\hline Tenosique & 59814 & 5857 & 9.8 & $\mathrm{C}$ \\
\hline Centro & 684847 & 31109 & 4.5 & $\mathrm{C}$ \\
\hline Jonuta & 30567 & 1335 & 4.4 & $\mathrm{E}$ \\
\hline Jalpa de Méndez & 87249 & 2862 & 3.3 & $\mathrm{E}$ \\
\hline Macuspana & 165729 & 12506 & 3.3 & $\mathrm{C}$ \\
\hline Balancán & 60516 & 1881 & 3.1 & $\mathrm{E}$ \\
\hline Teapa & 58523 & 1689 & 2.9 & $\mathrm{E}$ \\
\hline Emiliano Zapata & 30637 & 684 & 2.2 & $\mathrm{E}$ \\
\hline Huimanguillo & 188792 & 2912 & 1.5 & $\mathrm{E}$ \\
\hline Jalapa & 38231 & 441 & 1.2 & $\mathrm{E}$ \\
\hline Cárdenas & 258554 & 2967 & 1.1 & $\mathrm{E}$ \\
\hline Cunduacán & 138504 & 1265 & 0.9 & $\mathrm{E}$ \\
\hline Comalcalco & 201654 & 1334 & 0.7 & $\mathrm{E}$ \\
\hline Paraíso & 94375 & 766 & 0.3 & $\mathrm{E}$ \\
\hline
\end{tabular}

Nota: Indicadores socioeconómicos de los pueblos indígenas de México (2015:112) disponible en https://www.gob.mx/cms/uploads/attachment/file/239924/05-cuadro-01.pdf 


\section{Journal of the Academy $\mid 215$ |}

\section{iii. Localidades indígenas}

En los municipios de Tabasco existe un total de 291 localidades indígenas de tres tipos: con población indígena, con presencia de población indígena y con población indígena dispersa. El primer concepto hace referencia a una localidad cuya población indígena es igual o mayor a $70 \%$ de la población total (tipo A), o bien a una localidad donde la población indígena oscile en un rango entre el $40 \%$ y el $60 \%$ de la población total (tipo B).

El segundo tipo de localidad, aquella con presencia de población indígena, señala que un número significativo de la población en términos absolutos es indígena y mayor a 5,000 (tipo C), o que la población indígena es significativa en la localidad, pero su número es menor a 5,000 (tipo D). El último de ellos indica que en la localidad habita una minoría de la población indígena en relación con el total poblacional (tipo E).

Con base en esto, y el análisis de los datos del último censo INEGI, se puede precisar que 55 localidades de Tabasco, pueden designarse como poblaciones indígenas, porque cumplen las características que se mencionaron con anterioridad. Estas están conformadas por 25 localidades de tipo A, que constituyen el $8.6 \%$ del total de localidades indígenas (LI), y 30 localidades de tipo B, que representan el 10.3\%. El yokot'an y el ch'ol son las agrupaciones lingüísticas con mayor número de localidades de este tipo. Se encuentran distribuidas principalmente en los actuales municipios de Tacotalpa, Nacajuca, Balancán, Macuspana, Tenosique y Centro.

Asimismo, Tabasco alberga 29 núcleos poblacionales que corresponde al tipo de localidades con presencia indígena. De estas, 26 son de tipo C y 3 son de tipo D, lo que representa el $8.9 \%$ y el $1 \%$ del total de LI, respectivamente. En este rango se pueden encontrar poblaciones de todas las agrupaciones lingüísticas de Tabasco, con excepción del náhuatl y del maya. Este tipo de poblaciones se localizan mayormente en los municipios de: Tacotalpa, Nacajuca, Balancán, Macuspana, Tenosique, Huimanguillo, Jalpa de Méndez y Centro (Villahermosa).

El resto de las localidades se encuentran dentro del tipo E, denominadas localidades con presencia de población indígena dispersa. Estas constituyen el tipo más común en el territorio 


\section{Journal of the Academy $|216|$}

tabasqueño y comprenden un total de 181, es decir, el 62.2\% ${ }^{4}$ del total de LI. Por ende, gran parte de los municipios de la zona poseen este tipo de localidades, exceptuando a Jalapa en la región de la sierra y a Paraíso en la región chontal.

\section{iv. Aspectos socioeconómicos}

En Tabasco, las comunidades indígenas o con presencia indígena, sufren de marginación y pobreza en su gran mayoría, como es común en México. En este apartado analizaremos en forma concisa las principales características que, en general, poseen las localidades indígenas o con presencia indígena, lo que podrá permitir tener una relación de lo social con lo lingüístico.

\section{Accesibilidad}

El primer rubro a evaluar es en cuanto a su accesibilidad y al aislamiento histórico que hubo, debido a las condiciones geográficas y a que en Tabasco la cultura del agua es esencial. Para acceder a sus regiones, 178 localidades cuentan con carreteras pavimentadas y 99 transitan por caminos de terracería.

El resto habita en poblaciones que no conectan a carretera y donde se debe caminar entre 1 y $20 \mathrm{~km}$ para llegar a la más próxima, por mencionar un ejemplo, los indígenas chontales de Boca Grande 2da. Sección en el municipio de Centla, tienen que realizar diariamente este largo recorrido. Asimismo, solo 180 localidades poseen servicio de transporte público que permite el acceso a otras poblaciones cercanas o a la cabecera municipal.

\section{Actividades productivas}

La actividad productiva de mayor demanda en 234 localidades es la agricultura de subsistencia, llamada así porque mediante ella obtienen los productos básicos para subsistir: maíz, calabaza, frijol, camote, yuca, macal, chile, chayote, limón, naranja, hierbas para la cocina, distintos tipos de quelites, entre otros. Las localidades restantes diversifican sus actividades económicas principales entre la cría de animales, la pesca, el aprovechamiento forestal, el comercio al por

\footnotetext{
${ }^{4}$ Existe un vacío de datos en la base del INEGI (2010), para 26 localidades con presencia de hablantes indígenas, por lo que la suma de los porcentajes descritos no alcanza a sumar el $100 \%$.
} 


\section{Journal of the Academy $|217|$}

menor, la industria manufacturera y la de construcción, así como a la extracción de hidrocarburos. La agricultura es esencial para la adscripción de lo indígena, un ejemplo son los ch'oles, quienes se definen como los milperos (Rojas 2019, Sosa 2019).

Dado que estas actividades son realizadas en su mayoría por el género masculino, la principal población económicamente activa en estas localidades la constituye este sector con un $96.3 \%$. Mientras que el género femenino constituye la principal población no económicamente activa con un $77.4 \%$.

\section{Servicios públicos}

Los servicios públicos con que cuentan las localidades indígenas en Tabasco son escasos y mayormente irregulares en las regiones donde se hablan lenguas indígenas. Uno de los más básicos del que se carece es el servicio de agua potable, es limitado en 22 localidades y el servicio de drenaje en 57. En las localidades que cuentan con agua potable, el servicio es poco y en algunas, como en las comunidades indígenas del municipio de Tacotalpa, suele ausentarse por días.

El servicio de recolección de basura únicamente está disponible en 58 localidades que representa solo el $20 \%$ del total. Esto hace que se sigan reproduciendo prácticas antiguas que contaminan el ambiente, el suelo y el agua como son la quema de desechos plásticos, la dispersión de la basura a cielo abierto y la acumulación de residuos en lugares no aptos para ello.

Aunado a estas carencias, se encuentra el alumbrado público y la construcción de calles. Del primero solamente gozan 96 localidades lo que explica, en parte, el creciente aumento de actividades ilícitas en estos lugares. Las calles no son comunes en el paisaje rural y se confirma por el hecho de que existan solo en 62 localidades. Usualmente suelen encontrarse caminos, andadores y callejones de tierra y, en el caso de las zonas serranas, es común el uso de escalinatas.

La salud pública es otro de los temas álgidos cuando se habla sobre las poblaciones indígenas. Aunque físicamente existen 136 clínicas o centros de salud construidos en el mismo número de localidades, la realidad es que la mayor parte de ellas se encuentran sin el equipo adecuado 


\section{Journal of the Academy $|218|$}

o sin personal de salud que labore efectivamente en ellos. Afortunadamente, existen parteras que atienden los casos de alumbramiento $\mathrm{y}$, en menor medida, curanderos que ayudan a restaurar la salud de la población. No obstante, existen complicaciones que para ellos es imposible atender por falta de preparación en el área, que muchas veces se complican por la falta de atención oportuna.

La población derechohabiente de servicios de salud en estas comunidades suma un total de 136 160 afiliadas principalmente al Seguro Popular (85\%). El resto se encuentran afiliados a instituciones públicas como el Instituto Mexicano del Seguro Social-IMSS (5.5\%), el Instituto de Seguridad y Servicios Sociales para los Trabajadores del Estado (ISSSTE) (3.1\%) o a organismos estatales como el Instituto de Seguridad Social del Estado de Tabasco (ISSET), Instituto de Seguridad Social del Estado de México y Municipios (ISSSEMyM), Instituto de Seguridad y Servicios Sociales de los Trabajadores del Estado de Zacatecas (ISSSTEZAC), Instituto de Seguridad y Servicios Sociales para los Servidores Públicos del Estado de Aguascalientes (ISSSPEA) o Instituto de Seguridad y Servicios Sociales de los Trabajadores del Estado de Sonora (ISSSTESON) (2.8\%). Por otra parte, 43524 pobladores no se encuentran afiliados a ningún servicio de salud.

\section{Educación}

En lo que concierne a la educación formal, hay que considerar el hecho de que los pueblos indígenas poco han sido atendidos por políticas educativas en Tabasco. Esto limita la existencia de escuelas pensadas para población étnicamente diferenciada. La más pequeña de las localidades tabasqueña registra tan solo dos habitantes, mientras que la más poblada registra 4613. De ahí que únicamente en 174 de estas poblaciones se cuente con instalaciones para la enseñanza de educación preescolar y primaria, 88 para nivel secundaria y 28 para preparatoria.

Con estos últimos datos se puede observar que la cobertura más amplia la tiene la educación básica, por lo que no es de extrañarse que el grado promedio de escolaridad terminada para las zonas indígenas se concentre en este nivel: 7.4 para hombres y 6.7 para mujeres. No obstante, 4127 niños de 3 a 12 años no asisten a la escuela y de estos 1712 no saben leer ni escribir. 


\section{Journal of the Academy $|219|$}

En cuanto a la educación secundaria, 30535 terminaron este nivel. De ellos el $48 \%$ fueron mujeres. Asimismo, 29907 personas estudiaron más allá del nivel básico hacia la preparatoria y la educación superior. De esta cifra, el 45\% fueron mujeres. Con todo, 14270 personas de 15 años y más son analfabetas, de las cuales el $63 \%$ son mujeres.

Esto muestra que muchos de los hablantes de lenguas indígena jóvenes que quieren seguir estudiando se topan con mayores dificultades a medida que avanzan en sus grados de estudio, no solo por la carencia de ofertas cerca de su comunidad de origen sino también por la necesidad de ayudar al sustento de la familia.

Cabe destacar la importancia que tiene para la investigación sobre lenguas indígenas en Tabasco, el nacimiento de la Universidad Intercultural del Estado de Tabasco. Desde su fundación en 2005 y hasta el presente ha generado investigaciones y proyectos en lenguas indígenas en sus tres unidades académicas. En la región ch’ol/zoque se encuentra la sede principal y dos recientes inauguradas -entre los años 2013 y 2014- en la región chontal de Villa Vicente Guerrero y Tamulté de las Sabanas, en los municipios de Centla y Centro respectivamente.

\section{Hogar y vivienda}

Para concluir este apartado se presenta información referente a los hogares y viviendas. El promedio de hijos vivos nacidos en ellos es de tres personas y se considera como jefe del hogar al padre en 33932 viviendas, aunque en 6864 la jefatura se adjudica a la madre. El total de viviendas habitadas en estas localidades es de aproximadamente 40894 en las que habitan en promedio 4.5 personas.

Del total de viviendas habitadas, 5021 poseen piso de tierra y 22331 disponen de dos habitaciones o más. 31651 tienen acceso a energía eléctrica y 23562 a agua potable. Otras 36 539 han instalado por lo menos un baño, pero solo 35928 se han beneficiado con un sistema de drenaje. Es decir, que aún existen 3427 viviendas que no disfrutan de ninguno de estos servicios. 


\section{Journal of the Academy $|220|$}

\section{v. Perfil sociolingüístico}

Como se mencionó en el apartado anterior, en Tabasco se hablan ocho lenguas indígenas, además de una oficial. El español es la lengua mayor (Lmay) con un 97\% de la población y también es la lengua oficial (o), como en el resto del país. Es en esta lengua en la que mayormente se imparte la educación formal (e) y constituye una materia (m) para el currículo escolar. En cambio, las lenguas indígenas son lenguas menores (Lmen), por cuanto constituyen solo el 3\% del total poblacional y muy pocas ocasiones son utilizadas como vehículo de transmisión de conocimientos o como materia en las escuelas oficiales.

Considerando el casi nulo estudio de las variantes que existen en Tabasco, podría decirse que todas son lenguas vernáculas (V). No obstante, para un poco más de la mitad de ellas se ha propuesto una normalización lingüística para su escritura, los trabajos coordinados por el Instituto Nacional de Lenguas Indígenas (INALI) a partir de 2010 en los estados con mayor densidad de HLI, permiten tener un panorama más claro sobre dichas variantes a nivel nacional, sin embargo, hasta ahora, en Tabasco se han realizado pocos trabajos, por lo que pueden considerarse lenguas estándar (E): ch'ol, zoque, tzeltal, maya y tzotzil. Las únicas excepciones son el chontal o yoykot'an, el náhuatl y el zapoteco.

Asimismo, todas ellas pueden considerarse como de grupo (g) debido a su uso para la comunicación en distintos espacios funcionales de una comunidad particular. Entre ellos, las ceremonias o ritos religiosos antiguos donde la lengua es empleada con estos fines (r).

Es necesario precisar que dentro de las lenguas que se usan en Tabasco, también suelen encontrarse lenguas internacionales (i) usadas para comunicarse con hablantes de otros países y que se enseñan como materia en las escuelas (m). Dentro de ellas podemos encontrar el inglés, alemán, francés, italiano, japonés, chino, hebreo, portugués y ruso. Así, con estos datos, la fórmula del perfil lingüístico de Tabasco quedaría de la siguiente manera:

Tabasco (2020): 18L=1Lmay $($ Eom $)+1$ Lmen $(\operatorname{Vgm})+2 \operatorname{Lmen}(\operatorname{Vg})+$ 1Lmen $(\mathrm{Egm})+4 \mathrm{Lmen}(\mathrm{Eg})+9$ (ie) 
La fórmula nos permite aseverar que en Tabasco para el año 2020: se hablan 18 lenguas conformadas por una lengua mayor de carácter oficial (español), que se sigue presentando como la oficial y que se enseña en las escuelas de todo el estado, una lengua menor vernácula no estandarizada de grupo, que se enseña como materia en algunas localidades (chontal); dos lenguas vernáculas menores no estandarizada usada en grupo (náhuatl y zapoteco), una lengua menor estandarizada de grupo que se enseña en algunas localidades como materia escolar ( $c h$ 'ol), cuatro lenguas menores estandarizadas de grupo (tzotzil, tzeltal, zoque y maya) y nueve lenguas internacionales que se enseñan en la escuela como lenguas extranjeras y que ya mencionamos líneas atrás.

Las sociedades bilingües ${ }^{5}$ y las relaciones de diglosia entre las lenguas, han sido comunes en Tabasco durante gran parte de su historia. En un primer momento, como hemos revisado, diversas culturas mesoamericanas (chontal (yokot'an, zoque, náhuatl y ch'ol) convergieron en estas tierras, favoreciendo el intercambio político, comercial y cultural. Para esto, era imprescindible que la mayor parte de la población lograra comunicarse en náhuatl, la lengua franca de la región, por ser esta el idioma oficial del imperio cultural dominante en Mesoamérica en el pasado; hay que recordar que hubo un proceso de nahuatlización que documentaron varios historiadores.

Esto da evidencia de la posición de prestigio que gozaba la lengua náhuatl en comparación con las restantes. Situación que, por una parte, exigía un bilingüismo individual y social coordinado (véase Appel y Muysken 1996), que permitió una competencia comunicativa igualitaria, entre la lengua materna y el náhuatl, no solo del hablante en particular, sino también de la colectividad a la que pertenecía. Para las lenguas restantes del territorio, en cambio, solo se requería un bilingüismo individual relativo en el que un hablante, generalmente el comerciante, lograba comunicarse en otra lengua sin dominarla por completo.

\footnotetext{
${ }^{5}$ El fenómeno lingüístico denominado como bilingüismo es común en espacios donde ha existido contacto entre lenguas y hace referencia a la capacidad lingüística del hablante para comunicarse en dos lenguas (véase Appel y Muysken 1996; Hamers y Blanc 1989).
} 


\section{Journal of the Academy $|222|$}

Con la llegada de los colonizadores, las sociedades bilingües indígenas continuaron coexistiendo en el territorio junto con el español. Este último, como era de esperarse, pasó a ocupar el lugar de la lengua de prestigio, los hablantes de las lenguas vernáculas fueron obligados a usarlo en todos los espacios funcionales. Es decir, se formaron hablantes y sociedades bilingües coordinadas que eran capaces de usar ambas lenguas en igualdad de condiciones. No así para los españoles quienes desconocían las lenguas indígenas, salvo por algunos frailes que, con fines de evangelización, lograron un bilingüismo relativo acorde a sus intereses.

Hoy en día, en las poblaciones indígenas solo el $0.8 \%$ de la población mayor a 3 años es monolingüe y se concentran principalmente en las zonas serranas de los municipios de Tenosique y Tacotalpa. El resto presentan un bilingüismo individual y social coordinado (español-lengua indígena) con distintos dominios funcionales de la lengua como el hogar $(94 \%)$, escuela $(33.7 \%)$, iglesia $(33 \%)$ y comercio $(28.9 \%)$.

Esto visibiliza una fuerte tendencia al desplazamiento de la lengua indígena por el español. Un estudio realizado por Escobar (2019) muestra que, al menos en las poblaciones ch'oles, existe una tendencia del 56\% al mantenimiento de la lengua, mientras que el $44 \%$ restante ya experimentan un proceso de desplazamiento. En ambos casos, hay diferencias notables precisamente en los dominios funcionales. Así, las poblaciones que tienden al mantenimiento de la lengua manifestaron un dominio funcional amplio impulsado desde el seno familiar, lo que ha desarrollado una actitud positiva del hablante hacia su lengua. En cambio, las que tienden al desplazamiento han perdido, o han disminuido drásticamente, los dominios funcionales de la lengua incluso hasta en el hogar donde solo es hablado por las personas mayores. Esto ocasionado mayormente por que los hablantes han generado una actitud negativa hacia la lengua, a raíz de los comportamientos discriminatorios enfrentados y la imposición del español como lengua de prestigio.

\section{CONCLUSIONES}

Tabasco posee un amplio pasado histórico en donde la diversidad lingüística y cultural es evidente. Sobre la época prehispánica como hemos presentado a través de datos obtenidos desde la arqueología y de las crónicas rescatadas por personajes locales tabasqueños, que los 


\section{Journal of the Academy $|223|$}

primeros encuentros culturales entre grupos étnicos tuvieron que ver con grupos mayas en relación con zoques de la región serrana situada al sur de la entidad, en los límites con Chiapas. Más tarde, durante el dominio náhuatl, existió presencia de grupos zoques, mayas y nahuas, quienes convergían en este territorio manteniendo relaciones comerciales estrechas. Estas relaciones, en las que el náhuatl se consideraba como la lengua de prestigio, dieron lugar a un bilingüismo individual y colectivo coordinado que subsistió por mucho tiempo.

Durante la conquista, la situación socio lingüística cambió drásticamente. El yokot'an siguió siendo la lengua natural de los habitantes de Tabasco y el náhuatl la lengua franca de la región. Asimismo, había un número importante de hablantes de popoluca y de zoque, que además dominaban la lengua natural y la lengua franca para fines comerciales. Resulta elemental tomar en cuenta que entre 1520 y 1579 como refiere Martínez Assad (2011), la población originaria se vio diezmada por las epidemias de viruela, sarampión y tifo. Es decir, el bilingüismo coordinado se siguió manteniendo entre las poblaciones de origen mesoamericano, quienes además debían aprender la nueva lengua de prestigio que les fue impuesta durante los siglos XVI y XVII: el español, pero tanto el proceso de colonización, como las fuertes epidemias que azotaron Tabasco, disminuyeron a la población originaria.

Adicionalmente, muchas de las actitudes de la población mestiza hacia lo indígena en Tabasco ha provocado que se vayan perdiendo mucho de los rasgos culturales de identidad indígena, induciendo a muchos pueblos y comunidades de origen mesoamericano, a adoptar el español como lengua impuesta y las costumbres occidentalizadas, causando un alto desplazamiento lingüístico y cultural en sus poblaciones. La mayoría de los Hablantes de Lenguas Indígenas (HLI) actualmente son adultos mayores, son muy pocas las localidades en los que la lengua indígena aún sigue siendo la lengua materna de los niños. Esto indica que la siguiente generación ya no continuará con la herencia lingüística y cultural de estos pueblos, esto resulta preocupante y es urgente establecer medidas y acciones.

En cuanto al rezago social, los datos indican que gran parte de estas poblaciones carecen de servicios públicos básicos, como el agua y la luz, además de que su accesibilidad es muy problemática ya sea por falta de transporte o por falta de carreteras. El recurso económico que se maneja es muy limitado y se visibiliza la falta de oportunidades para la mujer, quien debe dedicarse exclusivamente a las labores del hogar en la mayoría de los casos. 


\section{Journal of the Academy $|224|$}

Si bien es cierto que el acceso a la educación básica ha crecido considerablemente con programas educativos para población indígena para el nivel primaria, no ha sido así para el resto de los niveles educativos. Los jóvenes y adolescentes indígenas regularmente deben salir de sus poblaciones para tener acceso al nivel medio superior y superior. Esto, con un presupuesto limitado y casi exclusivo para subsistir, es un gran problema para este sector quienes finalmente optan por formar una familia desde edades muy tempranas y dedicarse a la agricultura de subsistencia, la fundación de la Universidad Intercultural del Estado de Tabasco en el año 2005, que cuenta actualmente con tres unidades académicas, en dos regiones culturales, la región ch'ol-zoque en el municipio de Tacotalpa y región chontal en el municipio de Centla y de Centro, representan una estrategia que a largo plazo pretende impactar en el bienestar y desarrollo comunitario y sustentable para los pueblos originarios en Tabasco.

Finalmente consideramos relevante mencionar que la economía precaria tabasqueña en regiones indígenas, da como resultado problemas sociales como pobreza extrema, migraciones, desempleo, altos índices de suicidios, alcoholismo y la drogadicción, que finalmente conllevan a conflictos y delincuencia al interior de estas poblaciones donde hay fuerte presencia de comunidades indígenas y donde las lenguas originarias se encuentran amenazadas. Para concluir, creemos necesario plantear la urgencia de realizar más investigaciones sociolingüísticas e históricas en y sobre Tabasco, entidad federativa olvidada en varios momentos por las políticas públicas y lingüísticas en México.

\section{REFERENCIAS BIBLIOGRÁFICAS}

Appel, R. y Pieter, M., (1996). Bilingüismo y contacto de lenguas. Barcelona: Ariel.

Brice, H. (1986) La política del lenguaje en México: de la colonia a la nación. México: Instituto Nacional Indigenista.

De Alfaro y Santacruz, M. (1994). Relaciones históricos-geográficas de la provincia de Tabasco. Tuxtla Gutiérrez: Universidad Autónoma de Chiapas.

Ciudad Ruiz, A. y Lacadena García-Gallo, A. (2001). Tamactún-Acalán: Interpretación de una hegemonía política maya de los siglos XIV-XVI. Journal de la société des américanistes [En línea], 87, 01.

De Vos, J. (1980). La paz de Dios y del Rey. La conquista de la Selva Lacandona (1525-1821). México: Fondo de Cultura Económica. 
Escobar, A. (2019). Consideraciones sobre el desplazamiento de la lengua ch'ol en Tabasco. Revista Antropológica, (6)9, Posgrado de Antropología, Universidad Nacional Autónoma de México.

Galán, F., y Navarro, S. (2020). Resignificación de la Región Sierra de Tabasco a partir de elementos históricos y políticas públicas de etnicidad. Clivajes. Revista de Ciencias Sociales, 0(13), pp. 118-149.

García, A. y Martínez. M., (2007) Ch’oles, pueblos indígenas del México contemporáneo, Comisión Nacional para el Desarrollo de los Pueblos Indígenas, México.

Hamers, J. y Blanc, M., (1989). Bilinguality and Bilingualism, Cambridge: Cambridge University Press.

Houston, S. D., Stuart, D. y Robertson, J., (1998). Disharmony in Maya Hieroglyphic Writing: Linguistic Change and Continuity in Classic Society, en Anatomía de una civilización: aproximaciones interdisciplinarias a la cultura maya, Andrés Ciudad, $\mathbf{M}^{\mathrm{a}}$ Josefa Iglesias, J. M. García, A. Lacadena y Luis T. Sanz, eds., pp. 275-296. Publicaciones de la SEEM, 4. Madrid: Sociedad Española de Estudios Mayas.

Indicadores socioeconómicos de los pueblos indígenas de México (2015:112) disponible en https://www.gob.mx/cms/uploads/attachment/file/239924/05-cuadro-01.pdf

Lastra, Y. (1992). Sociolingüística para hispanoamericanos. Una introducción. México: El Colegio de México.

Martínez, C. R (2011). Breve Historia de Tabasco. El Colegio de México. México: Fondo de Cultura Económica.

Maza García del Alba, M. (2012). Cosmovisión de los Itzáes del Petén en el siglo XVIII. Tesis de licenciatura. Universidad Nacional Autónoma de México.

Moreno, C. (2014). La población en la provincia de Tabasco durante el periodo colonial (siglos XVI-XVIII). Un estudio revisionista, en Naveg @ mérica, revista electrónica editada por la asociación española de Americanistas (en línea), n. 13.

Rivet, P. (1954.) The maya cities. New York: London Elek Books, G. P. Putnam's.

Rojas V. (2019). Los ch’oles de Tabasco. La reconfiguración histórica y cultural de una región. Ruta Antropológica, 6(9), pp. 10-45.

Ruz, M. H. (1991). Los linderos del Agua. Francisco Montejo y los orígenes del Tabasco colonial. Villahermosa, Gobierno del Estado de Tabasco.

Ruz M. H. (2014) La sierra, una memoria fragmentada, en Tabasco serrano, miradas plurales, Mario Humberto Ruz (editor) UNAM-Gobierno del Estado de Tabasco, pp. 239-307. 


\section{Journal of the Academy $|226|$}

Salazar F (2014) Los pueblos de la sierra y los territorios de su región -los fundamentos del siglo XVI- en Tabasco serrano, miradas plurales, Mario Humberto Ruz (editor) UNAMGobierno del Estado de Tabasco, pp. 129-237.

Sosa E. (2019) Agricultura tradicional y apropiación del territorio: el caso de los milperos en Tacotalpa Tabasco. Ruta Antropológica, 6 (9) pp. 75-103.

Scholes, F. y Roys, R. (1996). Los chontales de Acalan-Tixchel. Universidad Nacional Autónoma de México, Centro de Estudios Mayas, Instituto de Investigaciones Filológicas.

Schumann, Otto. (1973) La lengua chol de Tila (Chiapas). México: Universidad Nacional Autónoma de México.

Voss, Alexander W. (2018). Presencia y distribución de la lengua maya yucateka en la península de Yucatán del clásico al posclásico tardío. en Cuadernos De Lingüística, de El Colegio De México, Número 2018.

Williamson R. (1986) El habla de Tabasco, estudio lingüístico. México: El Colegio de México. 